\title{
Hemodialysis catheters: An overlooked source of fibrin sheath endocarditis
}

\author{
Ismael Valle, Miguel Colon
}

\begin{abstract}
In recent years, healthcare associated infective endocarditis has shown an increasing trend most likely due to risk factor such as hemodialysis and diabetes. Staphylococcus aureus has become one of the most common causative organisms. We present the case of a 27-year Puerto Rican woman with diabetes mellitus and end stage kidney disease on hemodialysis who came to our Instruction with progressive dyspnea and a flu like illness with one week of evolution. Upon arrival to Emergency Department, a chest $X$-ray was performed revealing bilateral lung infiltrates and consolidates consistent with bilateral pneumonia. Empiric therapy for pneumonia was started but after seventy-two hours of antibiotic therapy patient continued deteriorating clinically. At that time, a chest computed tomography was performed, being remarkable for bilateral septic emboli and a large hyperattenuated lesion on the right atrium. Then, a transesophageal echocardiogram (TEE) was performed, since SVC and right atrium were unable to be properly assessed by transthoracic echocardiogram (TTE). The TEE revealed a large vegetation extending from superior vena cava to right atrial wall. Surgical management was then provided, in which a seven-centimeter vegetation was removed. Cephalosporin therapy was continued for six weeks leading to an uneventful
\end{abstract}

Ismael Valle ${ }^{1}$, Miguel Colon ${ }^{2}$

Affiliations: ${ }^{1}$ Department of Internal Medicine, San Juan City Hospital, Puerto Rico; ${ }^{2}$ Department of Infectious Disease, Auxilio Mutuo Hospital, Puerto Rico.

Corresponding Author: Ismael Valle, MD, San Juan City Hospital, Bo.Monacillos, San Juan PR 00936, Puerto Rico; Email: javyvalle@gmail.com

Received: 21 January 2018

Accepted: 05 February 2018

Published: 28 February 2018 recovery. Fibrin sheath formation secondary to hemodialysis catheter has become a frequent source of endocarditis that is overlooked by TTE. In view of the latter, we recommend TEE as the main approach to diagnose fibrin sheath endocarditis.

Keywords: Cephalosporin therapy, Infective endocarditis, Staphylococcus aureus, Transesophageal echocardiogram

\section{How to cite this article}

Valle I, Colon M. Hemodialysis catheters: An overlooked source of fibrin sheath endocarditis. Int $\mathrm{J}$ Case Rep Images 2018;9:100890Zo1IV2018

Article ID: 100890Zo1IV2018

$* * * * * * * * *$

doi: 10.5348/10089oZo1IV2018CR

\section{INTRODUCTION}

Infective endocarditis is defined as pathological evidence of microorganisms demonstrated by culture or histological examination of a vegetation, a vegetation that has embolized or intracardiac abscess alongside clinical evidence proposed by Modified Duke Criteria [1]. It is related to 40,000 to 50,000 new cases each year in the United States [2], being the most worrisome aspect, the increasing trend in health care associated factors, such as in hemodialysis patients [3, 4]. Staphylococcus aureus has been described as the most common causative organism of health care associated endocarditis including hemodialysis patients [1-5].

In the last decade and so, there are some cases reported $[6,7]$ among patients on hemodialysis with long term tunneled central venous catheters with associated 


\section{EDORIUM Journals}

fibrin sheath formation leading to a non-valvular source of infective endocarditis. The most common presentation of this entity is the formation of fibrin sheath vegetation in the superior vena cava $[7,8]$, that can be overlooked by transthoracic echocardiography (TTE) $[6,7,9]$. Thus, transesophageal echocardiography (TEE) has been suggested as the initial diagnostic approach in such cases $[6,9]$.

We report a case of a young female patient with end stage kidney disease on hemodialysis who presents with progressive dyspnea associated to a flu like illness who was initially admitted under the presumptive diagnosis of community acquired pneumonia and was later diagnosed with infective endocarditis secondary to a fibrin sheath vegetation.

\section{CASE REPORT}

A 27-year-old Puerto Rican woman with Diabetes Mellitus Type I and End Stage Kidney Disease (ESKD) on hemodialysis (HD) presented to our institution after developed widespread diffuse pain and progressive dyspnea associated to one episode of vomiting and diarrhea. On presentation, blood pressure was $80 / 50 \mathrm{mmHg}$, heart rate was 101 beats/min, respiratory rate was 28 respiration/min and a body temperature was $38.3^{\circ} \mathrm{C}$. Upon examination, tachycardia and bilateral lung crackles more prominent on left side were noted. There were no murmurs, rubs or gallops nor jugular venous distention (JVD).

Pertinent, laboratory results included hemoglobin of 9.0g/dL, hematocrit of $26 \%$, platelet count of 125,000/ $\mu \mathrm{L}$, and white cell count of $11,500 / \mu \mathrm{L}$. Blood urea nitrogen was $70 \mathrm{mg} / \mathrm{dl}$, creatinine was $5.8 \mathrm{mg} / \mathrm{dL}$ and potassium was $3.2 \mathrm{mEq} / \mathrm{L}$. Initial chest-X-ray (CXR) revealed bilateral patchy interstitial infiltrates suggestive of multilobar pneumonia (Figure 1). Then, empiric antibiotic therapy was started and patient was admitted. On the day of admission blood and sputum cultures were drawn.

After 72 hours of administration of antibiotic therapy, patient continue having multiple bouts of body temperature $>38.3^{\circ} \mathrm{C}$, along complains of worsening shortness of breath. A chest CT scan was performed revealing bilateral pulmonary septic embolism and a right atrial hyperattenuated lesion (Figure 2). At the same time, blood culture and sputum culture results were remarkable for growth of gram positive cocci in clusters. In view of the aforementioned findings on CT and blood cultures a transthoracic echocardiography was performed and a catheter on the right atrium was noted but no abnormalities associated to it were reported. Thereafter, a transesophageal echocardiogram (TEE) was performed. Superior vena cava was properly interrogated, revealing large vegetation which projects from the superior vena cava into the right atrial wall (Figure 3). Hemodialysis catheter was subsequently removed and patient was started on antibiotic therapy for Methicillin Sensitive Staphylococcus aureus (MSSA). Since patient had persistent bacteremia in spite of antibiotic therapy directed to MSSA, Thoracic Surgery evaluation was requested.Hence, thoracotomy procedure was recommended. An approximately, $7 \mathrm{~cm}$ vegetation was removed (Figure 4) from the superior vena cava and after six weeks of Cephalosporin therapy, resolution of symptoms was achieved.

\section{DISCUSSION}

Infection associated to hemodialysis catheters is a serious complication which occurs in more than half of catheters placed [10]. As previously mentioned, a great number of tunneled catheter infections are related to Staphylococcus aureus, [1-5], leading to infective

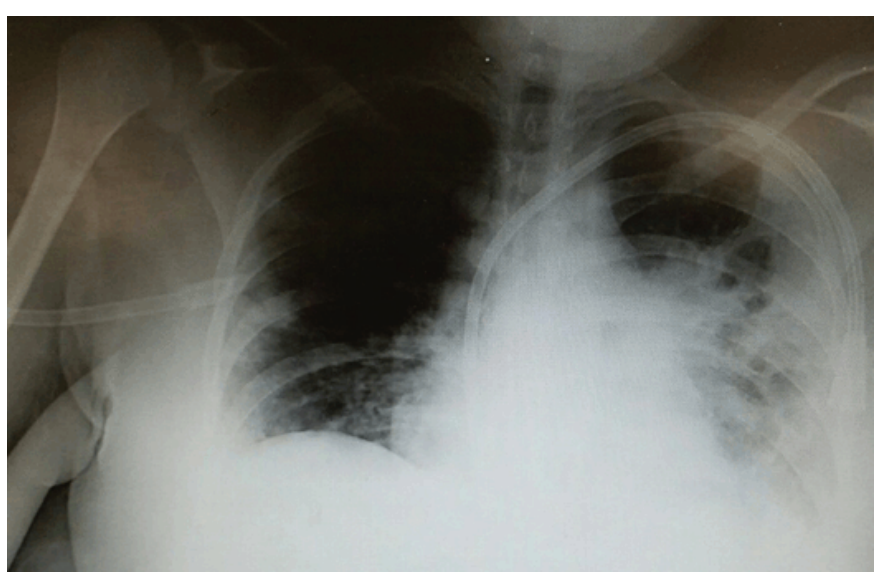

Figure 1: (A) Antero-posterior chest X-ray (CXR) showing a left subclavian tunneled dialysis catheter projecting into the right atrium (blue arrow) and (B) Lungs with bilateral interstitial infiltrates (yellow arrows).

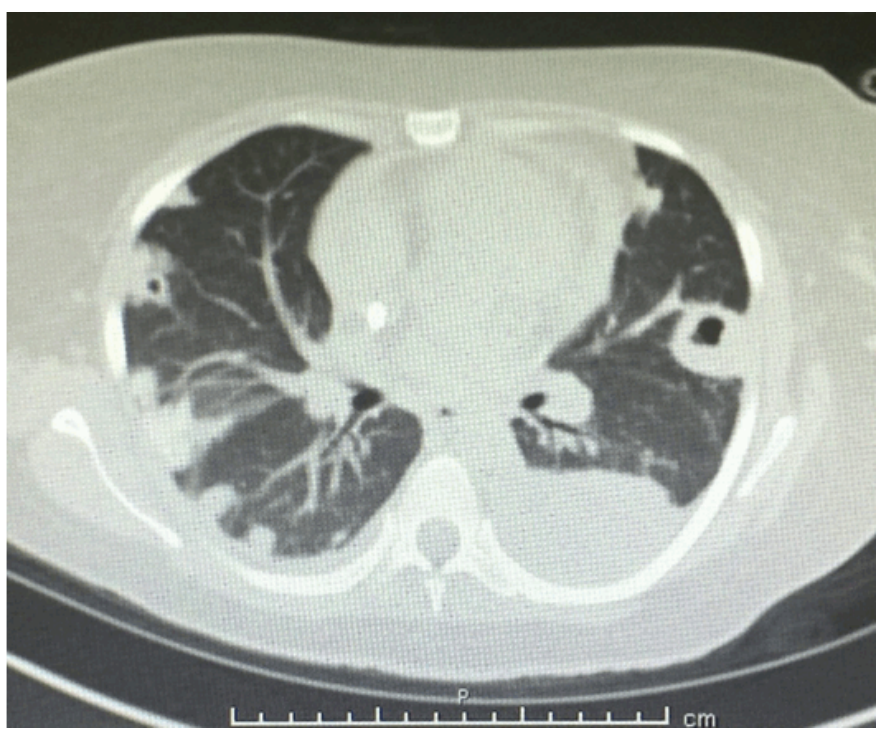

Figure 2: (A) Chest CT scan showing bilateral septic emboli (red arrow) and (B) Right atrium hyperattenuated mass like lesion with irregular contour (blue arrows). 


\section{EDORIUM Journals}

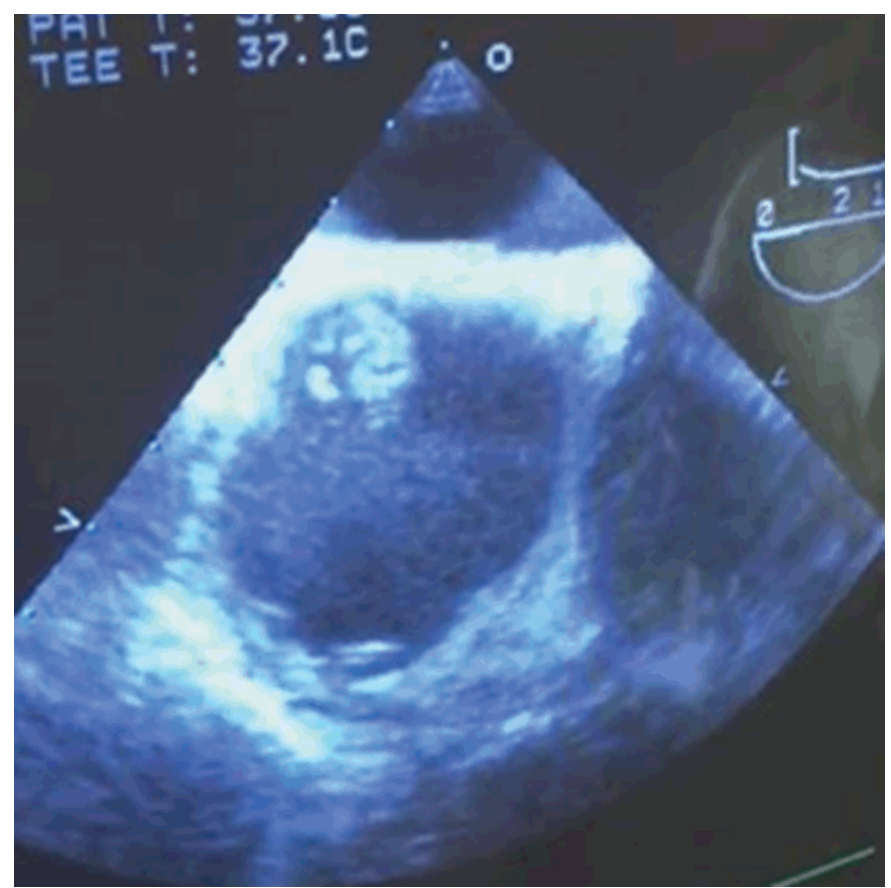

Figure 3: Transesophageal echocardiogram bicaval view showing a protruding mass like lesion into the right atrial chamber, suggestive of a large vegetation.

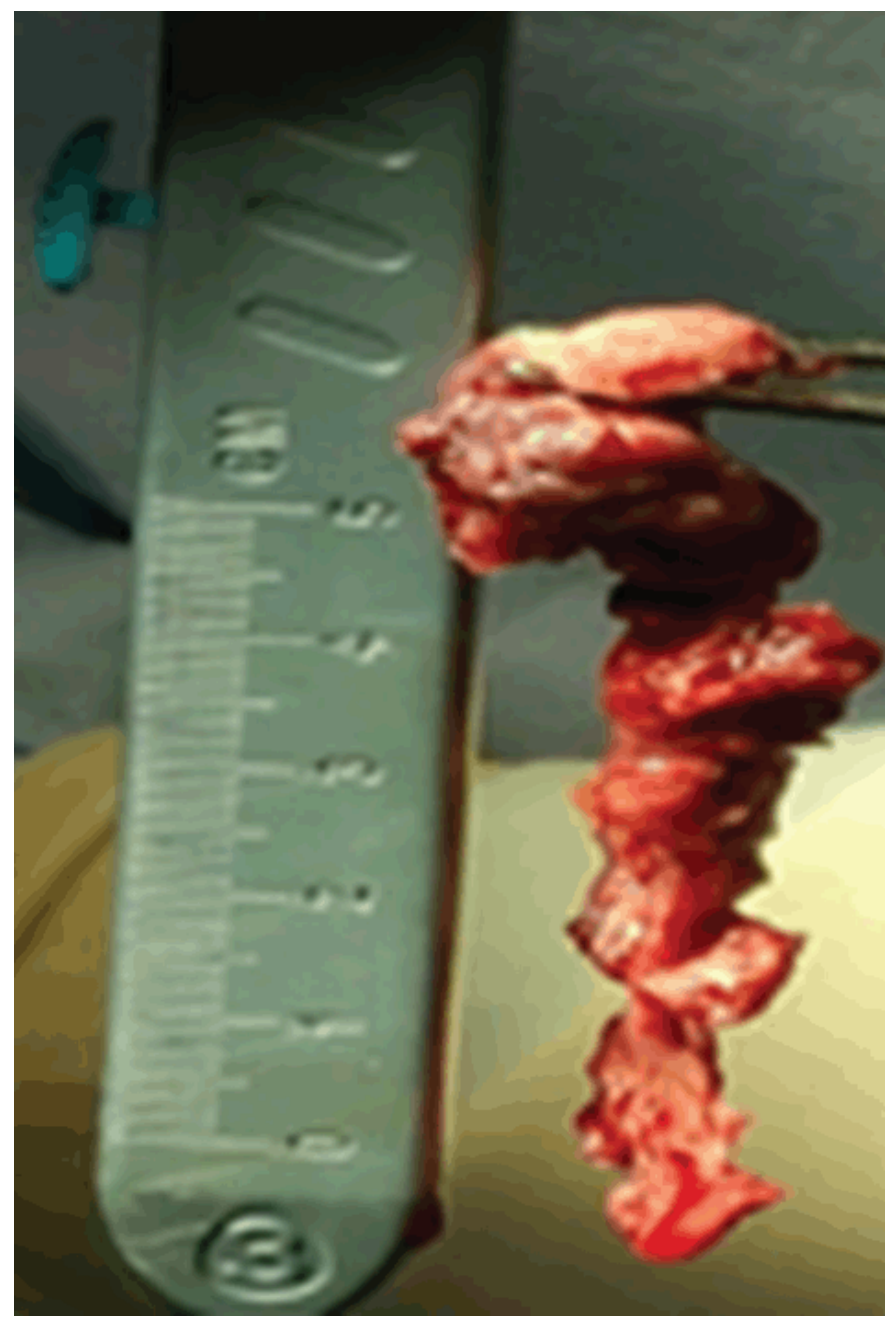

Figure 4: Fibrin sheath vegetation of approximately $7 \mathrm{~cm}$ extracted from the superior vena cava. endocarditis. The latter, is associated with higher mortality rates in patients on hemodialysis than in the general population [11], for which a prompt approach to make the aforementioned diagnosis can be lifesaving.

When central venous catheters are placed venous thrombosis and fibrin sheath formation might occur [12-14]. Sometimes these two entities can be overlooked since a proper interrogation of SVC is not possible with TTE, as occurred with our patient $[6,9]$. In such cases, TEE emerges as the appropriate diagnostic tool $[7,15]$. Once, these entities are detected, catheter removal should be encouraged $[6,13]$. In some cases, as occurred with our patient also, fibrin sheath can lead to vegetation formation serving as a source of infective endocarditis and persistent bacteremia [6, 9]. In view of this clinical scenario, our patient was taken to the Operation Room (OR) by the Thoracic Surgeon and a $7 \mathrm{~cm}$ vegetation was successfully excised. After the course of six weeks patient received antibiotic therapy with complete resolution of bacteremia. Recovery from surgery was uneventful.

Fibrin sheath formation in central veins secondary to tunneled hemodialysis catheters are associated with infection, aside from being a source of thrombus formation. In recent years, there are reports that provides enough evidence that fibrin sheath formation in the SVC is a source of non-valvular infective endocarditis. Using transthoracic echocardiography can overlook aforementioned findings in SVC, as occurred in our case.

Our case showed the importance of the TEE as an early diagnostic tool in regards to vegetations associated to fibrin sheath formation as a source of non-valvular infective endocarditis. At the same time, it offers a different management approach in such cases, in which thoracotomy procedure can be employed to eradicate the source infection. However, this must be examined in basis of each case.

\section{CONCLUSION}

We proposed that TEE should be used as an initial approach and the goal standard diagnostic tool, to warranty a detailed interrogation of the SVC. This would help physicians to make an accurate diagnosis and confer guidance in the management approach as well, whether it is surgery, or medical therapy alone, in order to directly impact mortality associated to infective endocarditis in patient on hemodialysis.

\section{REFERENCES}

1. Baddour LM, Wilson WR, Bayer AS, et al. Infective endocarditis in adults: Diagnosis, antimicrobial therapy, and management of complications: A scientific statement for healthcare professionals from the American heart association. Circulation 2015 Oct 13;132(15):1435-86. 
www.ijcasereportsandimages.com

2. Bor DH, Woolhandler S, Nardin R, Brusch J, Himmelstein DU. Infective endocarditis in the U.S., 1998-2009: A nationwide study. PLoS One 2013;8(3):e60033.

3. Cabell CH, Jollis JG, Peterson GE, et al. Changing patient characteristics and the effect on mortality in endocarditis. Arch Intern Med 2002 Jan 14;162(1):90-4.

4. Hoen B. Infective endocarditis: A frequent disease in dialysis patients. Nephrol Dial Transplant 2004 Jun;19(6):1360-2.

5. Selton-Suty C, Célard M, Le Moing V, et al. Preeminence of Staphylococcus aureus in infective endocarditis: A 1-year population-based survey. Clin Infect Dis 2012 May;54(9):1230-9.

6. Tang S, Beigel R, Arsanjani R, Larson B, Luthringer $\mathrm{D}$, Siegel R. Infective endovascular fibrin sheath vegetations-A new cause of bacteremia detected by transesophageal echocardiogram. Am J Med 2015 Sep;128(9):1029-38.

7. Sheikh MA, Shokr M, Ibrahim W, Cardozo S. Fibrin sheath-associated endovascular infection of the heart: The Trojan horse of indwelling central venous catheters. BMJ Case Rep 2017 Apr 17;2017. pii: bcr2016-219060.

8. Thakar S, Janga KC, Tolchinsky T, et al. Superior vena cava and right atrium wall infective endocarditis in patients receiving hemodialysis. Heart Lung 2012 May;41(3):301-7.

9. Peters PJ, Sohn J, Butler M, Okorie N, Moss EG, Corbett B. Retained fibrin sleeve: Transesophageal echocardiographic observations. J Am Soc Echocardiogr 2009 Jan;22(1):105.e1-2.

10. Schon D, Whittman D. Managing the complications of long-term tunneled dialysis catheters. Semin Dial 2003 Jul-Aug;16(4):314-22.

11. Nori US, Manoharan A, Thornby JI, Yee J, Parasuraman R, Ramanathan V. Mortality risk factors in chronic haemodialysis patients with infective endocarditis. Nephrol Dial Transplant 2006 Aug;21(8):2184-90.

12. Alomari AI, Falk A. The natural history of tunneled hemodialysis catheters removed or exchanged: A single-institution experience. J Vasc Interv Radiol 2007 Feb;18(2):227-35.
13. Santilli J. Fibrin sheaths and central venous catheter occlusions: Diagnosis and management. Tech Vasc Interv Radiol 2002 Jun;5(2):89-94.

14. Oguzkurt L, Tercan F, Torun D, Yildirim T, Zümrütdal A, Kizilkilic O. Impact of short-term hemodialysis catheters on the central veins: A catheter venographic study. Eur J Radiol 2004 Dec;52(3):293-9.

15. Freih M, Saba S. Superior vena cava (SVC) thrombosis: Diagnosis and follow up by transesophageal echocardiography (TEE). J Am Soc Echocardiogr 2005 Mar;18(3):280-1.

$$
* * * * * * * * *
$$

\section{Author Contributions}

Ismael Valle - Substantial contributions to conception and design, Drafting the article, Final approval of the version to be published

Miguel Colon - Substantial contributions to conception and design, Acquisition of data, Revising it critically for important intellectual content, Final approval of the version to be published

\section{Guarantor of Submission}

The corresponding author is the guarantor of submission.

\section{Source of Support}

None

\section{Consent Statement}

Written informed consent was obtained from the patient for publication of this case report.

\section{Conflict of Interest}

Authors declare no conflict of interest.

\section{Copyright}

(C) 2018 Ismael Valle et al. This article is distributed under the terms of Creative Commons Attribution License which permits unrestricted use, distribution and reproduction in any medium provided the original author(s) and original publisher are properly credited. Please see the copyright policy on the journal website for more information.
Access full text article on other devices

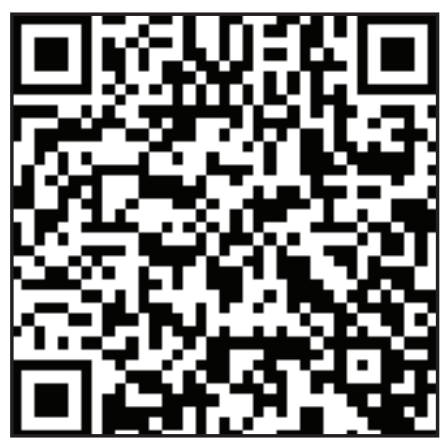

Access PDF of article on other devices

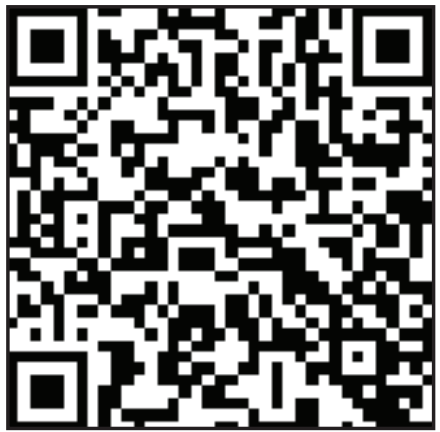

